\title{
Discovering Collaboration and Knowledge Management Practices for the Future Digital Factory
}

\author{
Myrna Flores ${ }^{1}$, Tomas Vera ${ }^{2}$, and Christopher Tucci ${ }^{2}$ \\ ${ }^{1}$ Research and Networking, Processes and IT, \\ CEMEX Global Center for Technology and Innovation, \\ CEMEX Research Group AG, Römerstrasse 13 CH-2555 Brügg, Switzerland \\ ${ }^{2}$ École Polytechnique Fédérale de Lausanne (EPFL), \\ College of Management, Chair in Corporate Strategy \& Innovation (CSI) \\ myrnafatima.flores@cemex.com, tomasvera@gmail.com, \\ christopher.tucci@epfl.ch
}

\begin{abstract}
Recently there has been an explosion of new technologies and tools such as wikis, blogs, tags, Facebook, among many others, that are commonly identified under Web 2.0 and which promise a new digital business ecosystem fed by formal/informal and internal/external relationships and interactions. Although Web 2.0 is very promising to enable such collective knowledge creation, technology by itself is not the only ingredient. It is also required to define the right strategy, governance, culture, processes, training, incentives among others, before implementing such innovative open spaces for collaboration and knowledge sharing. Therefore, the objective of this paper is to present a Knowledge Management (KM) Framework and a Maturity Model developed by a CEMEX and EPFL collaborative research project to discover the AS-IS collaboration practices in CEMEX before the implementation of the SMARTBRICKS Web 2.0 prototype for Business Process Management (BPM), currently under development by the Intelligent Manufacturing Systems (IMS) Swiss Digital Factory (DiFac) project.
\end{abstract}

Keywords: Collaboration Best Practices, Web 2.0, Knowledge Management Framework, Digital Factory.

\section{Introduction}

The Intelligent Manufacturing System (IMS) Digital Factory (DiFac) collaborative research project aims to develop a strategic planning design tool that is capable of supporting the construction of a digital factory. The Swiss IMS DIFAC project covers the workpackage dealing with the Enterprise Resource Planning (ERP), proposing a new Industrial Engineering System that should not only integrate information and knowledge from the production process but the complete Product Life Cycle [1].

To accomplish this objective, research targets the testing, implementation and diffusion of novel ICT technologies such as Semantic Web, RFID and discrete event simulation. CEMEX Research Group (CRG) is partner of the Swiss DIFAC project and leads Workpackage 1, which aims to develop a Web 2.0/Semantic Web prototype 
called SMARTBRICKS, currently under development, in collaboration with the University of Applied Sciences of Southern Switzerland (SUPSI). This new tool will enable CEMEX employees to share information and knowledge by exploiting Web 2.0 and semantic web technologies for Business Process Management (BPM). In other words, BPM specialists will evolve from "passive consumers" of business processes contents to "active contents developers" and accelerate BPM best practices global diffusion and sharing [2].

Although SMARTBRICKS is a very promising tool to enhance collaboration in BPM within CEMEX, it is also important to consider the organizational, cultural and change management elements for its successful implementation. Therefore a collaborative research project was defined and carried out by CEMEX Research Group and the College of Management of the École Polytechnique Fédérale de Lausanne (EPFL) to develop a Knowledge Management Framework and Maturity Model to assess current collaboration strengths and opportunities in CEMEX before implementing SMARTBRICKS. Therefore, the following sections will briefly explain the research outcomes of such CEMEX - EPFL collaborative project developed from September 2008 to February 2009.

\section{Research Methodology}

This CEMEX - EPFL collaborative research project had the following objectives:

1) To design a Generic Knowledge Management (KM) framework with its corresponding maturity level and

2) To discover current collaboration best practices in CEMEX applying the proposed KM Framework and maturity model through the development, deployment and analysis of an e-survey to potential users of the SMARTBRICKS tool from Processes and IT in CEMEX.

The LEAD methodology, developed in CEMEX Research Group [3], which proposes four stages to manage research projects with external partners (Learn, Energize, Apply and Diffuse) was applied in this collaborative research project with EPFL as follows:

\section{1) LEARN}

- The research project objectives were defined. CEMEX and EPFL agreed on the expected deliverables and deadlines.

- Extensive literature review on knowledge management, existing maturity models and Web 2.0 was carried out. Initially, relevant papers on the topic were provided by CEMEX to EPFL's project team. This latter complemented the literature review during the first two months.

- A glossary was developed to define all the important concepts linked to the project. Such document was continuously updated during the complete project.

\section{2) ENERGIZE}

- An initial Knowledge Management (KM) framework was proposed by EPFL. Afterwards, based on a several brainstorming sessions and CEMEX suggestions and requirements, a second and multilayered KM Framework was created by CEMEX and EPFL teams. 
- $\quad$ EPFL and CEMEX designed a questionnaire based on the agreed final KM Framework for its validation and to discover current KM practices.

- A sample of 60 employees working in Processes and IT in CEMEX was identified and invited to fill in the questionnaire in the form of an e-survey.

- An e-survey developed in SurveyMonkey and was filled in by 21 persons of the selected sample from the Processes and IT department within CEMEX to discover such AS-IS collaboration best practices and identify opportunity areas.

- A Knowledge Management Maturity Assessment Model was developed

\section{3) APPLY}

- The e-survey data was analyzed and applying the proposed maturity model, it was possible to map the key strengths and weaknesses and obtain an overall understanding in which level is currently CEMEX in terms of collaboration practices for knowledge management.

\section{4) DIFFUSE}

- During this last phase, diffusion of results are carried out within CEMEX, in scientific journals and international conferences

\section{Knowledge Management (KM) Foundations}

Back in 1959, Peter Drucker coined the term "knowledge worker" as one who develops and uses new knowledge in the workplace [4]. Even if the concept was developed already five decades ago, only in the last years organizations have started to recognize "knowledge" as a strategic intangible asset. In this context, firms require to understand how useful knowledge can be successfully shared, stored, reproduced and reused in a collective way for processes optimization.

For many, knowledge has become one of the most important resources a company disposes [5]. Researchers in knowledge management contend that any firm's competitive advantage depends on what it knows, how it uses what it knows, and how fast it can know something new [6]. But although creating knowledge is an important activity, knowledge has to be harnessed and leveraged to be useful. Learning occurs when knowledge in one part of an organization is transferred effectively to other parties and re-used to solve problems or to provide new and creative insights. Therefore knowledge sharing and reusing is what a "Learning Organization" is all about and is the vision of the future Digital Factory. In fact, Senge [7] defined a learning organization as a place where people continually expand their capacity to create the results they truly desire, where new and expansive patterns of thinking are nurtured, where collective aspiration is set free, and where people are continually learning to learn together.

But what is Knowledge and Knowledge Management (KM)? There is not one unique definition for either of them. Following Kogut and Zander [8], knowledge could be understood as recipe that specifies how to carry out activities. Devenport and Prusak [9] define knowledge "as a fluid mix of framed experiences, values, contextual information and expert insight that provides a framework for evaluating and incorporating new experiences and information. It originates and is applied in the minds of 
knowers. In organizations, it often becomes embedded not only in documents or repositories, but also in organizational routines, processes, practices and norms".

In regards to the Knowledge-based theory of the firm, it was developed by Winter [10], Kogut and Zander [8], [11], Nonaka [12], Nonaka and Takeuchi [13], Grant [14], and many other authors have contributed for its diffusion and continuous evolution. Wiig [15] states that KM is the management of corporate knowledge that can improve a range of organizational performance characteristics by enabling an enterprise to be more "intelligent active". Marshall [16] referred to KM as the harnessing of "intellectual capital" within the organization. According to Seemann et. al [17], Knowledge Management can be thought of as the deliberate design of processes, tools, structures, etc, with the intent to increase, renew, share, or improve the use of knowledge represented in any of the three elements (structural, human and social) of intellectual capital. For CISCO [18], Knowledge Management refers to a range of practices used by organizations to identify, create, represent, and distribute knowledge for reuse, awareness and learning across the organization.

One key element for successful Knowledge Management initiatives within a company is the definition and implementation of a knowledge management (KM) strategy and at the same time its diffusion is a requirement to make it possible to promote best practices, encourage employees to share their knowledge and collaborate, instigate managers to value those who do, and thus, finally increase and improve this valuable intangible asset. In fact, Zack [19], after doing research about KM in more than 25 firms has concluded that the most important context for guiding knowledge management is its firm's strategy. A few years later [20], Zack added that "once the role between strategy and knowledge is defined, the other aspects of strategic management such as resources allocation and organization design, among others, can be configured to bolster knowledge strengths and reduce knowledge weaknesses". As one interesting example, Hansen et. al [21], have identified two main strategies followed by business consulting firms: codification and personalization. The first one focuses on the codification of knowledge in repositories for it to be reused, while the second one in based on the development of new know-how and enabling it to be shared based on peer-to-peer interactions. The authors argue that firms can develop their strategy based on the customers they serve, the economics of its business and the people it hires.

\section{Assessing Existing Knowledge Management Frameworks and Maturity Models}

Although it is quite diffused that Knowledge Management can provide productivity returns, still many organizations struggle to identify which is the best possible path to implement a Knowledge Management strategy. Several consulting experts and academics have provided different models to enable the implementation of a KM strategy.

A model is a simplified representation of reality, developed with a specific goal; a maturity model follows the evolution of an entity from its early beginnings to a final status, be it one of declination and death or an ideal one of excellence and continuous improvement. Maturity models are usually structured on stages that have to be overcome one at a time, by fulfilling a set of requisites, or may allow for continuous improvement. Most of them have proved to be useful to measure the current maturity of 
the process employed, evaluate the current status of the field practices, set an objective for process design endeavors, guide the evolution of organizational change, and allow comparisons or benchmarking with similar efforts of other units or companies [22]. In order to develop a new Knowledge Management Framework for the future Digital Factory, this section will briefly explain the state of the art analysis of recently developed KM models and maturity models.

Gartner [23] has proposed a 5 level maturity model for collaboration ranging from no maturity to pervasive use of collaboration tools. According to a Gartner's research project carried out in 2007, most companies are around Level 2, where collaboration process maturity is emerging but still very new. Companies at this stage have realized that the basic tools are not enough; they are experimenting with new tools in order to make collaboration easier. However, only early adopters do any collaborative work, if at all. What Gartner provides is a model to support organizations to self-assess themselves and identify their maturity level. Even if the model is very complete, its weakness relies that it mainly focuses on IT collaboration tools and their adoption company wide. As a consequence, this maturity model is not enough to completely asses a company's standing point on collaboration as a whole, considering also soft aspects as the firm KM strategy, the organizational culture, recognition, etc.

On the other hand, SAP business analysts have developed a model that allows measuring knowledge management and collaboration practices in a more general manner. SAP provides a 5 level model [24], from initial to leader, but in contrast with the Gartner model, the SAP model is a more complete maturity assessment model, as it not only evaluates collaboration tools, but also states six different key areas that can be analyzed: People, Governance, Process, Content, Infrastructure and Tools/Techniques. The People improvement path, according to SAP, involves decreasing emphasis on only individual contributions but instead growing reliance on leveraging knowledge generated by communities; also increasing the recognition that the knowledge life cycle is woven into the fabric of daily work. While the Governance improvement path means increasing support for a culture that encourages knowledge sharing and validation of best practices. The "Process improvement path" involves improving content management and increasing the use of a collaborative environment in all communities, both internal and external. While the "Content path" means growing recognition of the importance of global reuse of knowledge assets. Finally, Infrastructure and Tools/Techniques paths deal with IT, as in the Gartner model.

McKinsey, on the other hand, states that Knowledge Management is not about managing knowledge but rather creating an environment that promotes and facilitates knowledge transfer [25]. As a consequence, the firm has implemented a four element model, where each element is multiplicative; in the sense that not delivering on any of them causes the knowledge transfer environment to fail. The four elements of the McKinsey model are: Clear Objectives, Knowledge Assets, Conducive Culture and Systems \& Infrastructure. Clear objectives means having concrete goals as to the business impact KM has to have in the company, e.g. economic results. Knowledge assets deals with the knowledge process itself, and if which contents are regarded as valuable assets by the company. A conductive culture is achieved through proper training, support and rewards for transferring knowledge between people. They highlight the importance of this element that is often ignored, while giving more attention to systems and infrastructure. In fact, most people often think only of the IT infrastructure when talking 
about KM. This model assigns a very high importance to culture, reducing SAP's proposed six key areas into a more manageable quantity. On the other hand, this KM model is not designed to evaluate collaboration maturity.

Besides these three previously described frameworks proposed by Gartner, SAP and McKinsey, other identified KM frameworks and maturity models are: KPMG [26], Infosys [27], Siemens [28], APQC [29], EFQM [30], among others [31], [32], [33], [34].

\section{Towards a Knowledge Management Framework and Maturity Model to Enable the Web 2.0 Future Digital Factory}

As a result of the previous analysis of existing KM frameworks and maturity models, there is a current need to create a new KM framework that includes all the key elements that can enable knowledge sharing and incorporate new Web 2.0 functionalities for Business Process Management (BPM). Therefore, the proposed KM framework targets the identification and assessment of collaboration practices to obtain the readiness level of the company before the SMARTBRICKS prototype implementation. It consists of several layers, built upon the central goals, and governed by the overall KM Strategy. This framework is shown in Figure 1.

Based on the proposed framework, a maturity model was also developed to assign maturity steps in each of the framework's elements, against which the present standing of the company can be evaluated. The final goal of this model is to provide a simple tool to assess the company's current maturity level in collaboration and knowledge management issues. It is important to assess maturity in order to identify strengths and weaknesses in order to establish a baseline to define objectives for improvement. This section will briefly describe the five levels of the proposed knowledge management self assessment tool as follows:

Level 1 - Initial: The organization doesn't have a strategy for KM. Each department, or even each individual works in his own fashion, there are no common tools or platforms and sharing is very limited, if existent at all. There are no established goals and there is no acknowledgement of the Knowledge Management (KM) Process. Even if some tools might be present, such as basic tools or infrastructure (phone, email system) they are not companywide and follow no clear strategy. Also, there is no expressed vision or clear objectives.

Level 2 - Basic: The company considers collaboration simply as communication; thus providing basic communication support. At this point, the company lacks clear goals concerning KM, though it might already have a vision of where it would like to be in the future. Tools and infrastructure are the items where the most emphasis has been made, at this maturity level. The knowledge process exists intrinsically, but it is not communicated to all employees, particularly the storage and sharing ones. Concerning support structures, there is no organizational design to improve knowledge transfer; rewarding systems are not in place and there is no time provided to allow employees to share documents, experiences, etc. However, at this stage, the basic infrastructure and tools are in place. Supervisors and employees are aware of the need 
of sharing information, but knowledge content is created individually, and there is no strong culture of collaboration, beyond superficial meetings.

Level 3 - Emerging: The company has started to realize how important KM is to remain competitive and increase its productivity and innovation outputs. It has already developed some companywide initiatives, especially in the Knowledge Enablers layer. The organization is experimenting on the many collaboration items and is trying to develop richer and more diverse forms of knowledge transfer. It has moved beyond basic communication, and is already doing some planning, contributing and creating collaboratively. The company realizes the necessity of properly storing the information. Even though the knowledge process is carried out, it is basically for explicit knowledge; little is being done to store and share the implicit knowledge of the individuals, beyond meetings with partial and incomplete minutes. There is some basic organizational design towards a more collaborative environment, but more effort has to be put into developing cross-functional work-groups. There are companywide available central repositories for information but of a basic nature, such as file servers. The company is already providing some integrated access to the knowledge repositories and is also starting to experiment with Web 2.0 tools, and some might be available for users. The collaboration culture is emerging, some colleagues collaborate and there are more standardized protocols, especially for explicit knowledge. The company already has a clearer vision of where it wants to be concerning $\mathrm{KM}$, there might also be some objectives. But no expected Business Impact has been analyzed, nor and Key Indicators been defined. There is no proper alignment of the various enablers towards the global KM Vision, since most of these processes are still being experimented with.

Level 4 - Expanding: The KM governance is formalized and understood by all employees in the company; a department is dedicated to the successful development of the KM initiative. Infrastructure and tools are carefully designed with collaboration as a goal, rather than as an experiment. Enablers have been optimized, and they are starting to be aligned with the overall KM strategy. Cross-functional tasks and teams are already being implemented. The company has significantly decreased its hierarchical nature. The Knowledge Management process has now been fully integrated companywide, at least for the explicit knowledge. Also, it has begun to be used to properly capture the more volatile implicit and tacit knowledge. Content is no longer created individually, but in a collective manner, by communities. A wide range of tools are available to search and explore the wide knowledge databases of the company. Information is properly organized, and therefore easy to access. Also, thanks to the implementation of Web 2.0 tools, such as tagging, finding the relevant information has become easier than ever. Infrastructure is mature. A collaboration-based rewarding system is in place although it is in an early stage. Employees, thanks to proper training, are developing the necessary skills and competencies to properly share and transfer knowledge. The collaboration culture is acknowledged as an influencing factor, and therefore as an enabler. The KM Strategy has been now properly laid out. The desired business impact of the KM Strategy has been established as well as the key indicators to measure the business impact. The company is beginning to align the various elements to its KM strategic views. 
Level 5 - Pervasive: The Knowledge Process is not only integrated inside the company, but also with external partners, such as suppliers, clients, consulting firms and Universities. The company has realized that knowledge is a strategic asset. Implicit, as well as explicit knowledge is successfully acquired, extracted, stored, shared and updates by everyone. Knowledge Enablers are not only optimized but are now fully aligned with the overall KM strategy. Processes are built around collaboration; a rewarding system is now fine-tuned and steadily encouraging collaboration. Every tool available to employees has been optimized and even social networking applications are being fully used, information is shared freely between colleagues and employees have completely integrated collaboration to their everyday work. A collaborative culture is fully internalized. The KM Strategy is already in place and is continuously maintained and updated according to changes in the company's internal and external environment. The KM Strategy has had a business impact which can be measured and felt throughout the company. All Knowledge Enablers are aligned properly to the global strategy.

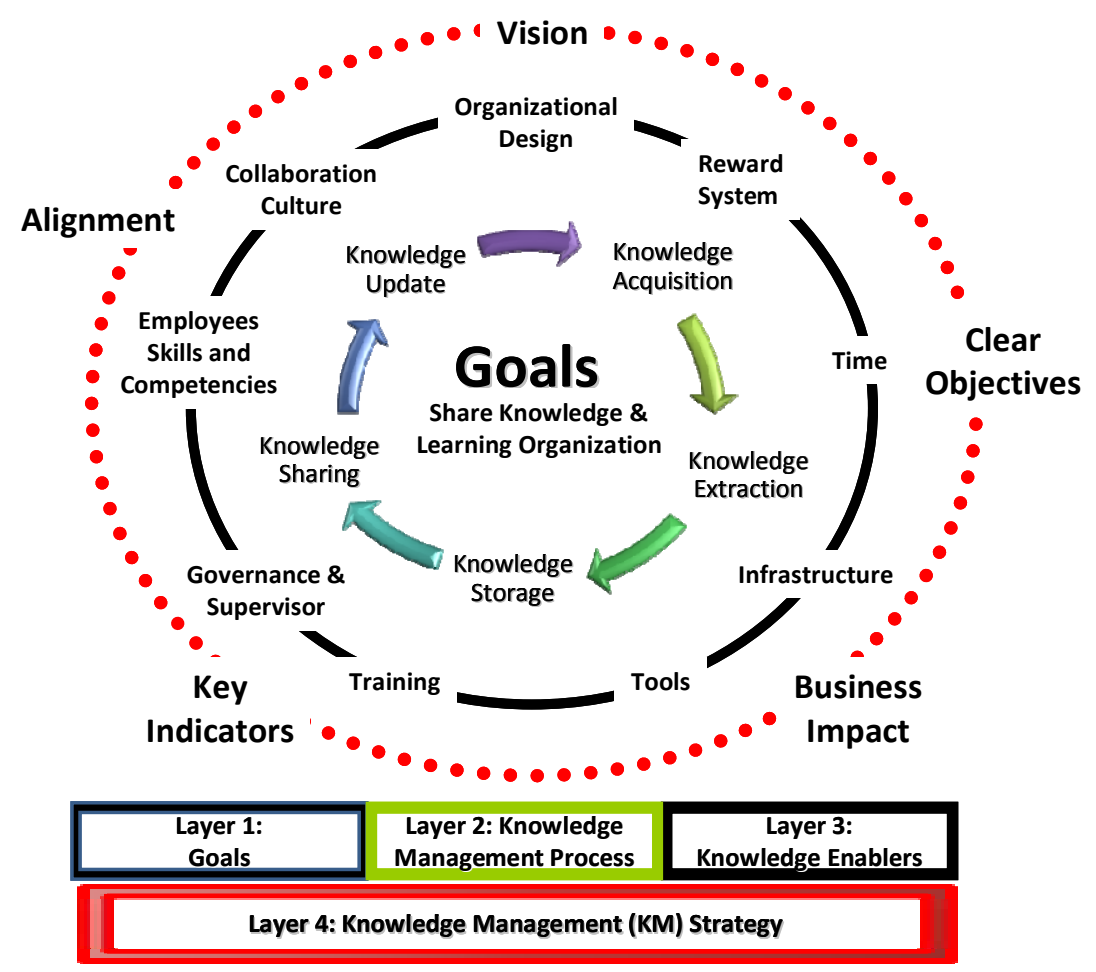

Fig. 1. Knowledge Management (KM) Framework for the Web 2.0 Digital Factory

\section{Conclusions}

A Knowledge Management framework and Maturity Self-assessment tool have been developed as a result of a CEMEX - EPFL collaborative research project. These latter 
have been applied in CEMEX to discover current collaboration and knowledge management practices to enable the future implementation of the SMARTBRICKS Web 2.0/Semantic Web tool currently under development by the Swiss DiFac project. For more information about the Digital Factory (DiFac), Intelligent Manufacturing Systems (IMS) Swiss project, please visit: www.difac.ch

Acknowledgments. The authors would like to thank the Swiss Innovation Promotion Agency - CTI International for the funding provided to the IMS Swiss Digital Factory (DiFac) Project. Gratitude is also given to Lorenzo Sommaruga and Junior Bontognali from the University of Applied Sciences of Southern Switzerland (SUPSI) for their effort to develop the SMARTBRICKS tool and for all their support during the IMS Swiss DiFac project. Special gratitude is also given to Ana Beatriz Dominguez Organero for the project's website development and communication activities carried out during the project.

\section{References}

1. Flores, M., Boër, C., Sommaruga, L., Salvadè, A., Lanini, M.: The Swiss IMS Digital Factory (DIFAC) project; implementing ICT technologies for sustainable innovation. In: Proceedings of the IFAC Workshop on Manufacturing Modelling, Management and Control (2007)

2. Flores, M., Sommaruga, L.: SMARTBRICKS: Developing an Intelligent Web tool for Business Process Management. In: International Conference of Concurrent Enterprising (ICE) Conference Proceedings, IMS Digital Factory (DiFac) Workshop, Leiden Holland (2009)

3. Flores, M.: The LEAD Research Methodology to manage collaborative open innovation projects. CEMEX internal document (2008)

4. Drucker, P.: The Coming of the New Organization. Harvard Business Review on Knowledge Management, pp. 1-19. Harvard Business School Press (1998)

5. Sveiby, K.E.: The New Organizational Wealth: Managing and Measuring KnowledgeBased Assets. Berrett-Koehler, San Francisco (1997)

6. Goh, S.C.: Managing effective knowledge transfer: an integrative framework and some practice implications. Journal of Knowledge Management 6(1), 23-30 (2002)

7. Senge, P.: The Fifth Discipline. Doubleday, New York (2006)

8. Kogut, B., Zander, U.: What Firms do? Coordination, Identity and Learning. Organization science (5), 502-523 (1996)

9. Devenport, T.H., Prusak, L.: Working Knowledge. Harvard Business School Press, Boston (1998)

10. Winter, S.G.: Knowledge and competences as strategic assets. In: Teece, D.J. (ed.) The Competitive challenge: Strategies for industrial Innovation and renewal. Ballinger, Cambridge (1987)

11. Kogut, B., Zander, U.: Knowledge of the firm, combinative capabilities, and the replication of technology. Organization Science 3, 383-397 (1992)

12. Nonaka, I.: The dynamic theory of organizational knowledge creation. Organization Science 5(1), 14-37 (1994)

13. Nonaka, I., Takeuchi, H.: The Knowledge-Creating Company. Oxford University Press, Oxford (1995) 
14. Grant, R.M.: Toward a Knowledge-based Theory of the firm. Strategic Management Journal, Winter Special Issue, 109-122 (1996)

15. Wiig, K.M.: Knowledge Management Foundations. Schema Press, Texas (1993)

16. Marshall, L.: Facilitating knowledge management and knowledge sharing: new opportunities for information professionals. Online 21(5), 92-99 (1997)

17. Seemann, P., DeLong, D., Stucky, S., Guthrie, E.: Building Intangible Assets: A Strategic Framework for Investing in Intellectual Capital. In: Second International Conference on the Practical Applications of Knowledge Management (PAKeM 1999), April 21-23 (1999)

18. CISCO, Knowledge Management - Ciscowiki, http://supportwiki.cisco.com

19. Zack, M.H.: Developing a Knowledge Strategy. California Management Review 41, 125 145 (Spring 1999)

20. Zack, M.H.: Rethinking the Knowledge Based Organization. MIT Sloan Management Review (Spring 2003)

21. Hansen, M.T., Nohria, N., Tierney, T.: What's Your Strategy for Managing Knowledge? Harvard Business Review (April 1999)

22. Trejo, J.M., Rodriguez, E.: A guided approach to Quality Improvement of Knowledge Management Practices. In: Proceedings of I-Know Conference, Graz, Austria (2006)

23. Gartner: Gartner's Maturity Model to Improve Enterprise Collaboration (2007)

24. SAP: A knowledge management maturity model for a global field services organization (2007)

25. Morosini, P., Denöel, E.: Knowledge Sharing at McKinsey \& Co., IMD Video (2001)

26. KPMG: Consulting, Knowledge Management Research Report (2000)

27. Infosys: Kochikar, V.P.: The Knowledge Management Maturity Model: A Staged Framework for Leveraging Knowledge. In: KMWorld 2000, Santa Clara, CA (2000)

28. Langen, M.: Knowledge Management Maturity KMMM® in Siemens AG. In: APQC Conference 2000 (2000)

29. O'Dell, C.: APQC Road Map to Knowledge Management Results: Stages of Implementa$\operatorname{tion}^{\mathrm{TM}}(2000)$

30. European Foundation For Quality Management: The EFQM Framework for Knowledge Management, European Foundation For Quality Management, Brussels (2005)

31. Natarajan, G., Ganesh, U.: Unleashing the Knowledge Force, Harnessing Knowledge for Building Global Companies. Tata McGraw Hill, New York (2007)

32. Hung, Y.H., Chou, T.S.C.: On constructing a knowledge management pyramid model. In: IEEE International Conference on Information Reuse and Integration (2005)

33. Haggie, K., Kingston, J.: Choosing Your Knowledge Management Strategy. Journal of Knowledge Management Practice (June 2003)

34. O'Dell, C., Grayson, C.J.: If only we knew what we know: identification and transfer of internal best practices. California Management Review 40(3), 154-174 (1998) 